\title{
Efficacy of preservative-free tafluprost in patients with normal-tension glaucoma previously treated with latanoprost
}

This article was published in the following Dove Press journal:

Clinical Ophthalmology

15 September 2014

Number of times this article has been viewed

\section{Matteo Sacchi \\ Edoardo Villani \\ Paolo Nucci}

University Eye Clinic, San Giuseppe Hospital, University of Milan,

Milan, Italy
Correspondence: Matteo Sacchi University Eye Clinic, San Giuseppe Hospital,Via San Vittore 12, 20I23, Milano, Italy Tel +390285994975

Fax +3902 294I 5945

Email matteosacchi.hsg@gmail.com

\section{Dear editor}

We read with interest the article entitled "Effects of tafluprost treatment for 3 years in patients with normal-tension glaucoma" by Inoue et al. ${ }^{1}$ In this study, the authors reported the efficacy of tafluprost in naïve patients with normal-tension glaucoma (NTG) over 3 years of follow-up.

The intraocular pressure (IOP)-lowering efficacy of tafluprost in NTG has been recently reported by Mizoguchi et $\mathrm{al}^{2}$ and Nakano et al. ${ }^{3}$

Whereas tafluprost is available in Europe in a preservative-free formulation, the three aforementioned studies ${ }^{1-3}$ were all carried out in Japan, where tafluprost is on the market as a benzalkonium chloride (BAK)-containing formulation.

Recent literature reported BAK-related ocular surface inflammation and epithelial damage, ${ }^{4}$ which led to the development of preservative-free antiglaucoma drugs. The cytotoxic effect of BAK on conjunctival and corneal epithelium has been claimed to increase ocular penetration of topical drug, enhancing the therapeutic effect. ${ }^{5}$

As the presence of preservative may play a role in the IOP-lowering effect, we believe it is of interest to report the efficacy of BAK-free formulations in NTG.

In a previous study by our group, BAK-free tafluprost showed a comparable efficacy to other prostaglandin analogs, with a safe profile in patients with primary open-angle glaucoma. ${ }^{6}$ In this letter, we want to report our experience with preservative-free tafluprost in patients with NTG. Herein, we present a retrospective study on 58 eyes of 58 patients (mean age $65.8 \pm 9.2$ years, range $43-91$ years) with NTG previously treated with BAK-preserved latanoprost who were switched to unpreserved tafluprost for ocular surface discomfort. The study was carried out with approval from the Institutional Ethics Committee (San Giuseppe Hospital, MultiMedica) and adhered to the tenets of the Declaration of Helsinki.

To be included, patients had to have unilateral or bilateral NTG. Diagnosis of NTG was made on the basis of optic-disk abnormalities and visual-field defects judged by a trained glaucoma specialist to be characteristic of glaucoma with IOP $<21 \mathrm{mmHg}$ at any time points of the diurnal IOP curve ( $8 \mathrm{am}, 11 \mathrm{am}, 2 \mathrm{pm}, 5 \mathrm{pm})$.

All patients were followed in our hospital (San Giuseppe Hospital) from January 2011 to December 2013. Inclusion criteria were: at least 3 months of treatment with BAK-preserved latanoprost followed by at least 3 months of treatment with unpreserved tafluprost; switch to tafluprost due to ocular discomfort; and availability of data from three diurnal IOP curves (untreated, treated with latanoprost, and after switch to tafluprost). 


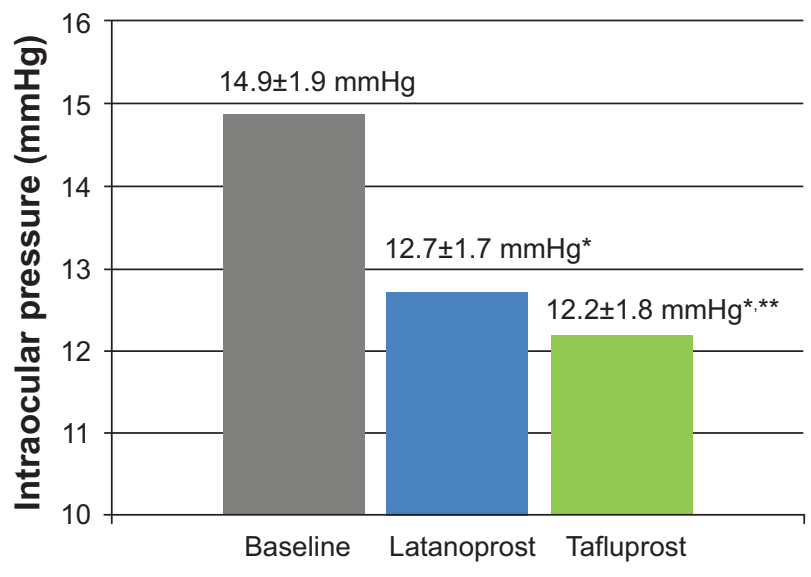

Figure I Intraocular pressure at baseline, with latanoprost, and after switch from latanoprost to tafluprost.

Notes: Data are shown as mean \pm standard deviation. $* P<0.001$, compared to baseline; $* * P=0.008$, compared to latanoprost.

Both latanoprost and tafluprost significantly reduced the diurnal mean IOP compared to baseline $(12.7 \pm 1.7 \mathrm{mmHg}$ versus $14.9 \pm 1.9 \mathrm{mmHg},-15 \%, P<0.001$ and $12.2 \pm 1.8 \mathrm{mmHg}$ versus $14.9 \pm 1.9 \mathrm{mmHg},-18 \%, P<0.001$, respectively) (Figure 1). Conjunctival hyperemia was reported in $17 \%$ and $8 \%$ of patients and fluorescein corneal staining in $6 \%$ and $2 \%$ of patients receiving latanoprost and tafluprost, respectively.

The retrospective nature of this study, the absence of a wash-out period, and the short follow-up are all limitations of this report and we are aware that our results need to be confirmed by powered, prospective, randomized clinical trials. However, to the best of our knowledge, this is the first report on the efficacy of BAK-free tafluprost in NTG.

In our study, the percentage IOP reduction from untreated baseline for both latanoprost (15\%) and tafluprost (18\%) was similar to that reported in other studies investigating the efficacy of prostaglandin analogs in NTG and ranging from $16 \%{ }^{7}$ to $19 \%{ }^{8}$

This study suggests the effectiveness of preservativefree tafluprost in NTG, showing results comparable to those obtained in Japanese patients ${ }^{1-3}$ using a BAK-preserved formulation.

\section{Disclosure}

The authors report no conflicts of interest in this work.

\section{References}

1. Inoue K, Tanaka A, Tomita G. Effects of tafluprost treatment for 3 years in patients with normal-tension glaucoma. Clin Ophthalmol. 2013;7:1411-1416.

2. Mizoguchi T, Ozaki M, Unoki K, Dake Y, Eto T, Arai M. A randomized crossover study comparing tafluprost $0.0015 \%$ with travoprost $0.004 \%$ in patients with normal-tension glaucoma [corrected]. Clin Ophthalmol. 2012;6:1579-1584.

3. Nakano T, Yoshikawa K, Kimura T, Suzumura H, Nanno M, Noro T. Efficacy and safety of tafluprost in normal-tension glaucoma with intraocular pressure of $16 \mathrm{mmHg}$ or less. Jpn J Ophthalmol. 2011;55(6):605-613.

4. Baudouin C. Detrimental effect of preservatives in eyedrops: implications for the treatment of glaucoma. Acta Ophthalmol. 2008;86(7):716-726.

5. Okabe K, Kimura H, Okabe J, et al. Effect of benzalkonium chloride on transscleral drug delivery. Invest Ophthalmol Vis Sci. 2005;46(2): 703-708

6. Ranno S, Sacchi M, Brancato C, Gilardi D, Lembo A, Nucci P. A prospective study evaluating IOP changes after switching from a therapy with prostaglandin eye drops containing preservatives to nonpreserved tafluprost in glaucoma patients. Scientific World Journal. 2012;2012:804730.

7. Quaranta L, Pizzolante T, Riva I, Haidich AB, Konstas AG, Stewart WC. Twenty-four-hour intraocular pressure and blood pressure levels with bimatoprost versus latanoprost in patients with normal-tension glaucoma. Br J Ophthalmol. 2008;92(9):1227-1231.

8. Mizoue S, Nakano T, Fuse N, Iwase A, Matsumoto S, Yoshikawa K. Travoprost with sofZia ${ }^{\circledR}$ preservative system lowered intraocular pressure of Japanese normal tension glaucoma with minimal side effects. Clin Ophthalmol. 2014;8:347-354. 


\section{Authors' reply}

Kenji Inoue'

Ayumi Tanaka'

Goji Tomita ${ }^{2}$

'Inouye Eye Hospital, 22nd Department of Ophthalmology,

Toho University School of Medicine, Tokyo, Japan

Correspondence: Kenji Inoue

Inouye Eye Hospital, 4-3 Kanda-Surugadai, Chiyoda-ku,

Tokyo I0I-0062, Japan

Email inoue-k@inouye-eye.or.jp

\section{Dear editor}

The authors would like to thank you for reviewing the present manuscript and for reading our previous paper on the effects of tafluprost in glaucoma patients. ${ }^{1}$ We would like to thank Dr M Sacchi for the valuable comments.

In patients with normal-tension glaucoma, the efficacy in decreasing intraocular pressure (IOP) and reducing corneal staining was preserved following a change in treatment from latanoprost with preservatives to tafluprost without preservatives. However, it remains unknown whether the concordance of the results obtained from the two different treatments was due to the change in the main agent (from latanoprost to tafluprost) and/or to the change in the use of preservatives in the eyedrops. Furthermore, discomfort was reported in patients enrolled in the treatment group, where latanoprost with preservatives was used. Therefore, we suggest that the increased adherence to the treatment was due to the use of eyedrops containing tafluprost without preservatives. Our opinions about products without preservatives are stated below.

The risks associated with the use of preservatives, particularly benzalkonium hydrochloride (BAC), include the appearance of adverse reactions such as hyperemia, allergy, and corneal epithelium disorder. ${ }^{2-4}$ These adverse reactions can contribute to a decrease in adherence to the treatment and a reduction in the efficacy of reducing IOP. A benefit of preservative use is the occurrence of a comfortable transition of eye drops to the intracameral increased. A more suitable study would compare the effects of medicated eyedrops with and without preservatives; however, tafluprost without preservatives was not available for patient treatment in Japan during the establishment of the study. ${ }^{1}$ Unit doses of tafluprost without preservatives became available in Japan in October 2013; therefore, we aim to subsequently compare the effects of tafluprost, both with and without preservatives, in a future study.
Latanoprost without preservatives (with formulation by filtration) has been available in Japan since 2010. We previously studied the effects of using latanoprost both with and without preservatives. ${ }^{5}$ Because this study was published in a Japanese journal, the results are currently only available in Japanese.

In our previous manuscript, ${ }^{5}$ three treatments were investigated: latanoprost with $\mathrm{BAC}$ was changed to latanoprost without $\mathrm{BAC}$; latanoprost without $\mathrm{BAC}$; and latanoprost without $\mathrm{BAC}$ was added to carteolol without BAC; each of the treatments were administered to NTG patients. The conclusion from the study was that IOP was maintained and adverse reactions of superficial punctate keratitis were weakened following 3 months of treatment in all cases in which latanoprost with BAC was previously used.

A translated version of the abstract from the Japanese manuscript is shown below.

Purpose: To investigate the ocular hypotensive effects and safety of latanoprost without benzalkonium hydrochloride (BAC).

Methods: Twenty patients with normal-tension glaucoma (monotherapy group), 14 patients who changed from latanoprost with BAC (changed therapy group), and 10 patients receiving added carteolol without BAC (additional therapy group). Intraocular pressure (IOP) and adverse reactions were checked at 1 and 3 months after treatment. In the changed-therapy group, superficial punctate keratitis was checked and compared before and after treatment.

Results: After 1 and 3 months, intraocular pressure decreased significantly in the mono and additional therapy groups, but after 3 months it remained unchanged in the changed therapy group. Because of adverse reactions, 2 patients $(4.5 \%)$ discontinued latanoprost without BAC. After 1 month, superficial punctate keratitis had improved in 6 patients $(42.9 \%)$ in the changed therapy group.

Conclusion: Latanoprost without BAC had strong hypotensive effects and was safely used in $95 \%$ of cases. ${ }^{5}$

In conclusion, eyedrops without preservatives are associated with a decrease in adverse reactions and improved adherence to the treatment. As such, we hope that the prescription and usage rates for these types of eyedrops increase in future clinical practice.

\section{Disclosure}

The authors report no conflicts of interest in this work. 


\section{References}

1. Inoue K, Tanaka A, Tomita G. Effects of tafluprost treatment for 3 years in patients with normal-tension glaucoma. Clin Ophthalmol. 2013;7:1411-1416.

2. Roslin LM, Bell NP. Preservative toxicity in glaucoma medication: clinical evaluation of benzalkonium chloride-free $0.5 \%$ timolol eye drops. Clin Ophthalmol. 2013;7:2131-2135.
3. Noecker R, Miller KV. Benzalkonium chloride in glaucoma medications. Ocul Surf. 2011;9(3):159-162.

4. Baudouin C. Detrimental effect of preservatives in eyedrops: implications for the treatment of glaucoma. Acta Ophthalmol. 2008;86(7):716-726.

5. Inoue K, Masumoto M, Wakakura M, Tomita G. [Ocular hypotensive effects and safety of latanoprost without benzalkonium hydrochloride]. Atarashii Ganka. 2011;28(11):1635-1639. Japanese.

\section{Publish your work in this journal}

Clinical Ophthalmology is an international, peer-reviewed journal covering all subspecialties within ophthalmology. Key topics include: Optometry; Visual science; Pharmacology and drug therapy in eye diseases; Basic Sciences; Primary and Secondary eye care; Patient Safety and Quality of Care Improvements. This journal is indexed on

\section{Dovepress}

PubMed Central and CAS, and is the official journal of The Society of Clinical Ophthalmology (SCO). The manuscript management system is completely online and includes a very quick and fair peer-review system, which is all easy to use. Visit http://www.dovepress.com/ testimonials.php to read real quotes from published authors. 